\title{
BACTERIOLOGICAL STUDY AND ANTIMICROBIAL SENSITIVITY PATTERN IN CASES OF OSTEOMYELITIS
}

\begin{tabular}{ll}
\hline Microbiology & \\
Dr Spriha Smriti* & MBBS,MD, Deptt of Microbiology PMCH, Patna. *Corresponding Author \\
\hline Dr Babita Kumari & MBBS,MD, Deptt of Microbiology PMCH, Patna. \\
\hline $\begin{array}{l}\text { Dr Pratulya } \\
\text { Nandan }\end{array}$ & Associate Professor, Deptt of Microbiology PMCH, Patna. \\
\hline
\end{tabular}

\begin{abstract}
Introduction- Osteomyelitis refers to infection of bone marrow which spread to the bone cortex and periosteum. Osteomyelitis is most commonly caused by pyogenic bacteria. Despite greater advances in treatment the management of osteomyelitis is quite challenging due to increasing development of antibiotic resistance.

Material And Methods - 100 patients were evaluated. Samples like pus or exudates or pieces of necrotic tissue were taken. Samples were subjected to gram's staining and culture. Antimicrobial sensitivity was done using Kirby Bauer disc diffusion method.

Results - Staphylococcus aureus was the most common organism isolated followed by E.coli, Klebsiella pneumoniae P. aeuroginosa and Proteus. Staphylococcus aureus + E coli was the most common polymicrobial organism isolated. In trauma cases most common organism isolated was Staphylococcus aureus. Gentamycin was the commonest antibiotic which was effective for treatment of osteomyelitis followed by amikacin, tobramycin and levofloxacin.In cases of gram positive organism max were seen to sensitive to vancomycin and linezolid.
\end{abstract}

\section{KEYWORDS}

Osteomyelitis, Staphylococcus aureus, Pyogenic, E.coli

\section{INTRODUCTION:}

Osteomyelitis refers to an infection of the bone marrow which may spread to the bone cortex and periosteum via the Haversian canals. It results in inflammatory destruction of the bone and if the periosteum become involved, necrosis occurs. When dead bone becomes detached from healthy bone, it is known as a sequestrum.

Osteomyelitis, an infection of bone is caused most commonly by pyogenic bacteria and mycobacteria. Osteomyelitis may be acute (2 weeks) or chronic ( $>6$ months duration) and can be further categorized into two main subgroups-

(a)Acute hematogenous Osteomyelitis

(b)Direct (Contagious Osteomyelitis).

Risk Factors- Trauma, prosthetic orthopaedic device, Diabetes, Alcoholism, Tuberculosis

Typical sites:-Vertebra (Adults $>$ children), long bone (Children>adult); and axial joints (Sternoclavicular, Sacroiliac Joints).

Types Of Osteomyelitis:

1. Pyogenic osteomyelits

2. Tuberculosis Osteomyelitis

3. Fungal Osteomyelitis

4. Coccidioidal Osteomyelitis

5. Actinomycotic Osteomyelitis

6. Blastomycotic Osteomyelitis

7. Cryptococcosis Osteomyelitis

8. Syphllitic Osteomyelitis

\section{MATERIALAND METHOD}

The present work will be conducted in the Department of Microbiology, Patna Medical College, Patna.

\section{Specimen Collection:-}

The specimens was collected from patients admitted in orthopaedic department of P.M.C.H, Patna. Specimens collected were pus or exudates or necrotic tissue. Samples were collected by using sterile cotton swab with all aseptic precautions. Samples were transported to the laboratory. 100 patients were evaluated through proper history and clinical examination standard protocol for proper identification of organism was done.

- Direct Gram's staining of sample for identification.

- Culture:-Collected pus, swab or exudate is inoculated on nutrient agar, mac conkey agar, blood agar and other as required and incubated at $37^{\circ} \mathrm{c}$ overnight aerobically. After $24 \mathrm{hrs}$ incubation of swabs on the following basis:-
Colour morphology+-colour, shape, size, opacity, surface, etc.Gram staining was done-either gm+ve or gm-ve.Motility test was done.Biochemical test was done for identification of organism. Antibiotic susceptibility testing was done by Kirby Bauer's Method on Mueller Hinton agar as per CLSI guidelines.

\section{RESULT}

100 Patients were evaluated who were admitted in orthopaedics department of PMCH, Patna. Sex incidence in the present study was 68 males to 42 female. Average age incidence in this study was $10-20$ Yrs. Locations of infection included foot $35(35 \%)$, leg $50(50 \%)$, hand in 5 $(5 \%)$ and spine $10(10 \%)$.The most common factor leading to osteomyelitis was trauma/accidents $(50 \%)$ followed by orthopaedic implants(35\%) and post surgical wound (15). In the present study 89 samples showed growth whereas 11 samples were sterile. Out of the 89 samples $61(61 \%)$ samples showed gram positive organism( 55 isolates of S.aureus and 6 isolates of CONS) and $39(39 \%)$ were gram negative organism( 26 isolates of E.coli followed by 8 isolates of Klebsiella, 4 isolates of Pseudomonas and 1 isolate of Proteus). Among the polymicrobial infection Staph aureus and E.coli was the most common. In the present study, organism was commonly sensitive to Gentamycin, Amikacin,levofloxacin,ofloxacin and tobramycin Majority of S.aureus species showed resistance to amoxicillin followed by cephalexin. Majority of strains were sensitive to vancomycin and linezolid.E.coli strain was mostly resistant to amoxicillin(100\%) followed by resistance to cefuroxime and cefotaxime.P.aeuroginosa showed max resistance to ciprofloxacin followed by piperacillin and cefipime.P. mirabilis was seen resistance to cefotaxime followed by ceftazidime and it was found $100 \%$ sensitive to imipenem.

\section{DISCUSSION}

Sex incidence in the present study was 68 males to 42 female. Wadkar Met al 2014 also reported higher incidence in males compared to females.Average age incidence in this study was $10-20$ Yrs which is near to that of Betler (1940) $90 \%$ of patient below the age of $20 y r s . K a u r ~ J$ et al (2008) showed lower extremity bones were involved in $60 \%$ of cases followed by upper extremity (14\%). Rest cases included short bones of hand and feet $(10 \%)$ and pevic bones $(8 \%) . \%)$.In the present study the most common factor leading to osteomyelitis was trauma/accidents $(50 \%)$ followed by orthopaedic implants (35\%) and post surgical wound (15). Wadekar DM et al(2014) reported most common factor for osteomyelitis was trauma(44\%) followed by post surgical infections $(23 \%)$ and orthopaedic implants $(21 \%)$. In the present study most common organism isolated was Staphylococcus aureus followed by enterobacteriaceae and P.aeuroginosa. Gilmore et al (2009) found nearly the same finding.

\section{REFERENCES}

1. Ananthanarayan and Paniker's textbook of Microbiology $9^{\text {th }}$ edition. 
2. Javreguui LE: Diagnosis \& management of bone infection Marcel Dekker, 1955.

Mollan R.A.B, Craig B.F and Biggant J.D:chronic Sclerosing osteomyelitis 666, 583:1984.

4. Shadling B: Acute hematogenous osteomyelitis 34:520,1960.

5. Trueta J.Acute hematogenous osteomyelitis its pathology and treatment 14:59, 1953

6. Wadekar MD, Anuradha K, Venkatesh D. Chronic osteomyelitis: aetiology and antibiotic susceptibility pattern. Int J Recent Trends in Science an Technology 2014;9(3):337-340

7. Gilmour WM. Acute hematogenous osteomyelitis. J Bone Joint Surg 2009(4): 841-851 